\title{
Qualidade do sono em pacientes pós-cirurgia cardíaca com diferentes índices de massa corporal
}

\author{
Sleep quality in patients in patients submitted \\ to coronary artery bypass grafting with different \\ body mass indexes
}

\author{
Graciele Machado Brandão' \\ Adriana Laiane de Carvalho Santos ${ }^{2}$ (1) \\ Amanda Lucas Pessoa Silva ${ }^{3}$ (1) \\ André Raimundo Guimarães 4 (1) \\ André Luiz Lisboa Cordeiro ${ }^{5}$
}

${ }^{1-3}$ Faculdade Nobre (Feira de Santana). Bahia, Brasil.gracybrandao1@hotmail.com, adrianasantos@gmail.com, amandasilva@gmail.com ${ }^{4}$ Instituto Nobre de Cardiologia (Feira de Santana). Bahia, Brasil. andremed@bol.com.br ${ }^{5}$ Autor para correspondência. Faculdade Nobre (Feira de Santana), Escola Bahiana de Medicina e Saúde Pública (Salvador). Bahia, Brasil. andrelisboacordeiro@gmail.com

RESUMO | INTRODUÇÃO: Evidências mostram que existe correlação positiva entre o Índice de Massa Corpórea (IMC) e a qualidade do sono. A avaliação de como o IMC pode influenciar a qualidade do sono de pacientes no pós-operatório pode ser útil para tomada de decisão relacionada ao tratamento fisioterapêutico. OBJETIVO: Comparar a qualidade do sono em pacientes pós-cirurgia cardíaca em diferentes IMC. MÉTODOS: Trata-se de um estudo transversal. Pacientes de ambos os sexos, com idade superior a 18 anos e submetidos a revascularização do miocárdio via esternotomia mediana e circulação extracorpórea foram incluídos. O Questionário do Índice de Qualidade do Sono de Pittsburgh, que mede a qualidade retrospectiva do sono, foi aplicado, na alta da Unidade de Terapia Intensiva (UTI), em pacientes de ambos os sexos agrupados por IMC em grupos eutrófico, sobrepeso e obesidade, para investigar as alterações no comportamento do sono pós-cirurgia cardíaca desses grupos. Pontuações de 0-4 indicam boa qualidade do sono e de 5-10 indicam qualidade ruim. Os dados foram expressos em média e desvio padrão. Para comparação entre os grupos o teste de ANOVA foi usado. Foi considerado como significativo um $p<0,05$. RESULTADOS: O estudo foi composto por 120 pacientes, com uma predominância do sexo masculino num total de $79(65,83 \%)$ pacientes e idade em torno de $60,22 \pm 1,34$ anos. As pontuações das variáveis desse questionário como Duração do sono (Grupo eutrófico:0, sobrepeso:0, obesidade:2, p=0,02), Distúrbio do sono (Grupo eutrófico:1, sobrepeso:1, obesidade:2, $p=0,01$ ) e Disfunção diurna (Grupo eutrófico:1, sobrepeso:1, obesidade:2, $p=0,04$ ) foram estatisticamente significativos. Comparando a pontuação geral entre os grupos eutrófico:2, sobrepeso:5 e obesidade:10 identificou-se que a qualidade do sono tende a piorar à medida que o IMC aumenta. CONCLUSÃO: Os pacientes que apresentavam um IMC maior, como do grupo obesidade, apresentaram pior qualidade do sono, enquanto os pacientes do grupo eutrófico possuíram melhor qualidade do sono.

PALAVRAS-CHAVE: Sono. Período Pós Operatório. Índice de Massa Corporal. Cirurgia Cardíaca.
ABSTRACT | INTRODUCTION: Evidence shows that there is a positive correlation between the Body Mass Index (BMI) and sleep quality. The assessment of how the BMI can influence the sleep quality of patients in the postoperative period can be useful for decision making related to physiotherapeutic treatment. OBJECTIVES: To compare the sleep quality in patients undergoing CABG at different BMI. METHODS: Patients of both sexes, aged 18 years or over and submitted to coronary artery bypass grafting via median sternotomy and cardiopulmonary bypass (CPB) were included. This is an observational study. The Pittsburgh Sleep Quality Index Questionnaire, which measures the retrospective sleep quality, was applied, upon discharge from the Intensive Care Unit (ICU), in patients of both sexes grouped by BMI into eutrophic, overweight, and obesity groups to investigate changes in sleep behavior post-cardiac surgery in these groups. The data were expressed as means and standard deviations. For comparison between groups, the ANOVA test was used. $\mathrm{P}<0.05$ was considered significant. RESULTS: The study consisted of 120 patients, with a predominance of males in a total of 79 (65.83\%) patients and age around 60,22 $\pm 1,34$ years. The scores of the variables in this questionnaire such as Sleep Duration (Eutrophic Group:0, Overweight:0, Obesity:2, $p=0.02$ ), Sleep Disturbances (Eutrophic Group:1, Overweight:1, Obesity:2, p=0.01) and Daytime Dysfunction (Eutrophic Group:1, Overweight:1, Obesity:2, $\mathrm{p}=0.04$ ) were statistically significant. Comparing the total score between the groups eutrophic:2, overweight:5, and obesity:10, it was found that sleep quality tends to worsen as the BMI increases. CONCLUSION: Patients with a higher BMI, as in the obesity group, had worse sleep quality, while patients in the eutrophic group had better sleep quality.

KEYWORDS: Sleep. Post-Operative Period. Body mass index. Cardiac surgery 


\section{Introdução}

A cirurgia cardíaca é considerada de grande porte e os pacientes submetidos a esse procedimento são encaminhados para uma Unidade de Terapia Intensiva (UTI) no pós-operatório (PO). Embora o monitoramento em UTI seja necessário para otimizar a recuperação, o tempo de internação pode prejudicar a qualidade do sono, por se tratar de uma unidade considerada geradora de estresse $\mathrm{e}^{1-3}$.

O estresse na UTI advém do grande número de equipamentos, profissionais e procedimentos que frequentemente interrompem o ciclo circadiano, causando prejuízos como o aumento da pressão arterial dos pacientes ${ }^{4}$. Além disso, problemas relacionados a feridas cirúrgicas, terapia medicamentosa, alterações do apetite, alterações emocionais e distúrbios neuromusculares podem levar a alterações na qualidade do sono dos pacientes no $\mathrm{PO}^{5}$.

Estudos têm demonstrado que a má qualidade do sono afeta a maioria dos indivíduos submetidos a procedimentos cirúrgicos, sendo a insônia o principal distúrbio relacionado. Portanto, o indivíduo que não dorme pode apresentar fadiga, cansaço, tensão, diminuição do desempenho intelectual, sintomas de depressão, ansiedade, sonolência diurna, dores musculares e irritabilidade ${ }^{6,7}$. Avaliar a qualidade do sono pode possibilitar ajustes terapêuticos relacionados à prescrição de exercícios físicos. Nesse contexto, a utilização do instrumento conhecido como Índice de Qualidade do Sono de Pittsburg (PSQI) ${ }^{8}$ tem se mostrado eficaz e capaz de fornecer informações quantitativas e qualitativas sobre a qualidade do sono ${ }^{9,10}$.

Vários estudos científicos mostram que há evidências de que o Índice de Massa Corporal (IMC) pode influenciar na qualidade do sono ${ }^{11-17}$. Madrid-Valero et al. ${ }^{16}$ relataram que a má qualidade do sono estava associada a um maior IMC. Para Hung et al. ${ }^{17}$, indivíduos com sobrepeso ou obesidade apresentam pior qualidade do sono do que aqueles com peso normal. Embora a literatura mostre que existe uma correlação positiva entre o Índice de Massa Corporal e a qualidade do sono, a quantidade de trabalho é escassa quando um terceiro descritor é adicionado como cirurgia cardíaca a essa correlação.
Dessa forma, este estudo tem como objetivo comparar a qualidade do sono em pacientes submetidos à cirurgia de revascularização do miocárdio com diferentes índices de massa corporal.

\section{Métodos}

\section{Desenho do Estudo}

Foi realizado estudo observacional com pacientes do Instituto Nobre de Cardiologia (INCARDIO), localizado na cidade de Feira de Santana, Bahia, no período de agosto de 2019 a janeiro de 2020. Foi aprovado pelo Comitê de Ética em Pesquisa da Faculdade Nobre de Feira de Santana, sob o número 4.008.896. CAAE (29839020.0.0000.5654). Todos os pacientes participantes assinaram o Termo de Consentimento Livre e Esclarecido (CIF).

\section{Critérios de elegibilidade}

Foram incluídos pacientes de ambos os sexos, com 18 anos ou mais e submetidos à cirurgia de revascularização do miocárdio por esternotomia mediana e circulação extracorpórea (CEC). Pacientes com doenças oncológicas, doenças infecciosas com repercussão no estado geral, endócrinas (diabetes mellitus e tireoide não tratada), pacientes em pós-operatório recente (até 60 dias de pós-operatório) foram excluídos de qualquer intervenção cirúrgica, pacientes com dificuldade de compreensão do questionário proposto, trazendo incompatibilidade de informações ou tendo incapacidade de responder à mesma pergunta, ou que se recusou a respondê-la.

\section{Protocolo do Estudo}

Após os pacientes atenderem aos critérios de inclusão, dados pessoais, história clínica, altura, peso e cálculo do Índice de Massa Corporal (IMC) foram calculados no período pré-operatório de cirurgia cardíaca. O cálculo foi realizado pela divisão do peso (em $\mathrm{Kg}$ ) por altura ao quadrado (em metros). Assim, dividimos os grupos em três classificações: eutrófico (IMC entre 18,5 a 24,9 kg / m2), sobrepeso (IMC entre 25 a 29,9 kg / m2) e obeso (IMC maior que $30,0 \mathrm{~kg} / \mathrm{m} 2$ ). Para informações sobre o comportamento do sono, foi aplica- 
do o Questionário do Índice de Qualidade do Sono de Pittsburgh (PSQI), que avalia a qualidade e os distúrbios do sono no período de um mês anterior à cirurgia. É composto por 19 questões de autorrelato e 5 questões dirigidas ao cônjuge ou companheiro de quarto.

Após todos os dados coletados e divididos os grupos, o questionário PSQI foi aplicado na alta da UTI. Verificamos as pontuações de cada questionário e analisamos as mudanças no comportamento do sono após a cirurgia cardíaca relacionada por cada grupo classificado.

\section{Instrumentos de pesquisa}

O questionário de Pittsburgh, originalmente desenvolvido por Buysse et al., , mede a qualidade retrospectiva do sono e distúrbios ao longo de um período de 1 mês para uso na prática clínica e em pesquisa. Ele discrimina entre bons e maus dormentes e fornece uma avaliação breve e clinicamente útil de vários distúrbios do sono.

É composto por 19 questões que são agrupadas em 7 componentes, cada um em uma escala graduada em pontuações de zero (nenhuma dificuldade) a três (dificuldade severa). Os componentes do PSQI são: C1 Qualidade subjetiva do sono, C2 Latência do sono, C3 Duração do sono, C4 Eficiência do sono usual, C5 Alterações do sono, C6 Uso de medicamentos, C7 Disfunção do sono diurno. A soma dos valores atribuídos aos sete componentes varia de 0 a 21 no escore total do questionário, indicando que quanto maior o número, pior a qualidade do sono. Pontuações de 0-4 indicam boa qualidade do sono, 5-10 indicam má qualidade e acima de 10 indicam distúrbios do sono.

\section{Análise estatística}

Para a análise dos dados, foi utilizado o Statistical Package for the Social Sciences (SPSS) versão 20.0. A normalidade foi avaliada pelo teste de KolmogorovSmirnov. Os dados foram expressos em médias e desvios-padrão. Para comparação entre os grupos, foi utilizado o teste ANOVA. $\mathrm{P}<0,05$ foi considerado significativo.

\section{Resultados}

Durante o período da pesquisa, foram admitidos 134 pacientes, dos quais 34 foram excluídos devido ao pós-operatório recente (4), dificuldade de compreensão do questionário proposto (20) e recusa em respondê-lo (10) (figura 1). O estudo foi composto por 120 pacientes, com predomínio do sexo masculino em um total de $79(65,83 \%)$ pacientes e idade média em torno de $60 \pm 1$ anos. A comorbidade mais prevalente foi Hipertensão Arterial Sistêmica (HAS), com 86 $(71,66 \%)$ pacientes e Índice de Massa Corporal (IMC) em torno de $27,3 \pm 4,5 \mathrm{~kg} / \mathrm{m} 2$. Os outros dados são mostrados na Tabela 1.

Figura 1. Fluxograma de elegibilidade do paciente

Avaliado para elegibilidade

$$
(n=134)
$$

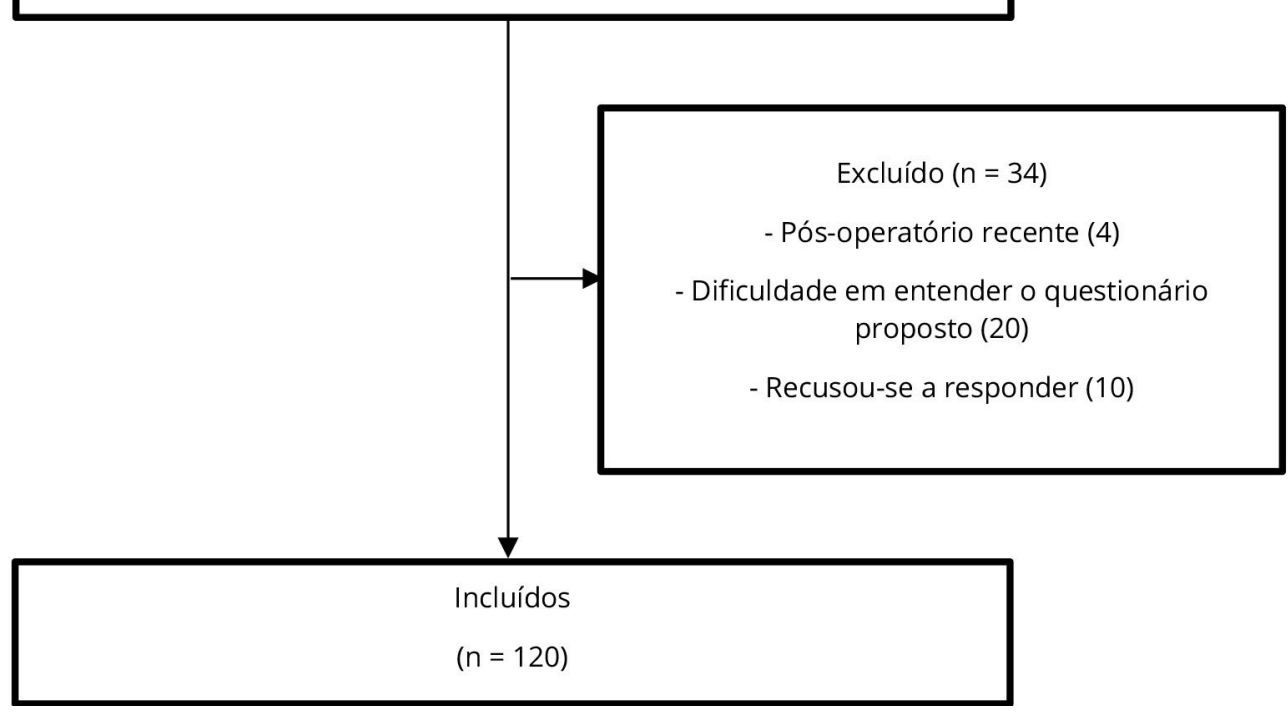


Tabela 1. Características clínicas dos pacientes estudados

\begin{tabular}{|c|c|c|c|c|}
\hline Variável & $\begin{array}{l}\text { Grupo eutrófico } \\
\qquad(n=31)\end{array}$ & $\begin{array}{l}\text { Grupo Sobrepeso } \\
(\mathbf{n}=55)\end{array}$ & $\begin{array}{c}\text { Grupo } \\
\text { Obesidade }(\mathrm{n}= \\
\text { 34) }\end{array}$ & Valor de $p$ \\
\hline Idade (anos) & $59 \pm 4$ & $62 \pm 5$ & $59 \pm 5$ & 0,64 \\
\hline \multicolumn{5}{|l|}{ Sexo } \\
\hline Masculino & $20(65 \%)$ & 37 (67\%) & $22(65 \%)$ & 0,11 \\
\hline Feminino & $11(35 \%)$ & $18(33 \% \%)$ & $12(35 \%)$ & \\
\hline IMC (kg/m²) & $22 \pm 3$ & $27 \pm 3$ & $33 \pm 2$ & $<0,01$ \\
\hline \multicolumn{5}{|l|}{ Comorbidades } \\
\hline HAS & $23(74 \%)$ & $39(71 \%)$ & $24(71 \%)$ & 0,83 \\
\hline DLP & $15(48 \%)$ & $22(40 \%)$ & $19(56 \%)$ & 0,35 \\
\hline DM & $12(39 \%)$ & $20(36 \%)$ & $15(44 \%)$ & 0,38 \\
\hline IAM & $3(10 \%)$ & $5(9 \%)$ & $4(12 \%)$ & 0,87 \\
\hline Sedentarismo & $14(45 \%)$ & $22(40 \%)$ & $16(47 \%)$ & 0,54 \\
\hline
\end{tabular}

IMC - Índice de Massa Corporal; HAS - Hipertensão Arterial Sistêmica; DLP - Dislipidemia; DM - Diabetes Mellitus; IAM - Infarto Agudo do Miocárdio.

Os pacientes de todos os grupos analisados submetidos à cirurgia cardíaca tiveram um tempo de circulação extracorpórea (CEC) de 91,32 $\pm 2,78$ minutos. No Tempo de Ventilação Mecânica (VM) dos pacientes foi em torno de $6,33 \pm 0,47$ horas. Os valores são mostrados na Tabela 2.

Tabela 2. Características cirúrgicas dos pacientes estudados

\begin{tabular}{ccccc}
\hline Variável & $\begin{array}{c}\text { Grupo eutrófico } \\
(\mathbf{n}=\mathbf{3 1})\end{array}$ & $\begin{array}{c}\text { Grupo Sobrepeso } \\
(\mathbf{n}=\mathbf{5 5})\end{array}$ & $\begin{array}{c}\text { Grupo } \\
\text { Obesidade } \mathbf{( n =} \\
\mathbf{3 4 )}\end{array}$ & Valor de $\mathbf{p}$ \\
\hline Tempo de CEC (min) & $89 \pm 12$ & $91 \pm 9$ & $94 \pm 11$ & 0,64 \\
\hline $\begin{array}{c}\text { Tempo de VM } \\
\text { (horas) }\end{array}$ & $6 \pm 2$ & $7 \pm 3$ & $6 \pm 3$ & 0,78 \\
\hline Número de pontes & $2 \pm 1$ & $2 \pm 1$ & $2 \pm 1$ & 0,93 \\
\hline Número de drenos & $2 \pm 1$ & $2 \pm 1$ & $2 \pm 1$ & 0,94 \\
\hline $\begin{array}{c}\text { Tempo de } \\
\text { clampeamento } \\
\text { (minutos) }\end{array}$ & $79 \pm 18$ & $85 \pm 15$ & $83 \pm 14$ & 0,53 \\
\hline $\begin{array}{c}\text { Tempo de cirurgia } \\
\text { (horas) }\end{array}$ & $231 \pm 24$ & $245 \pm 19$ & $255 \pm 21$ & 0,11 \\
\hline
\end{tabular}

CEC - Circulação Extracorpórea; VM - Ventilação Mecânica.

A Tabela 3 mostra a pontuação dos grupos de pacientes estudados no questionário de Pittsburg que avalia a qualidade do sono. Percebe-se que variáveis como duração do sono (grupo eutrófico: 0, grupo com sobrepeso: 0 , grupo obesidade: $2, p=0,02$ ), distúrbio do sono (grupo eutrófico: 1 , grupo com sobrepeso: 1 , grupo obesidade: $2, p=0,01$ ) e disfunção diurna (grupo eutrófico: 1, grupo com sobrepeso: 1, grupo obesidade: $2, p=0,04$ ) foram estatisticamente significativas. Ainda é possível verificar que entre os escores dos grupos de pacientes, o escore geral foi 10 no grupo obesidade, indicando pior qualidade do sono. 
Tabela 3. Escore do questionário de Pittsburg entre os grupos estudados

\begin{tabular}{ccccc}
\hline Variável & $\begin{array}{c}\text { Grupo eutrófico } \\
(\mathrm{n}=31)\end{array}$ & $\begin{array}{c}\text { Grupo Sobrepeso } \\
(\mathrm{n}=55)\end{array}$ & $\begin{array}{c}\text { Grupo } \\
\text { Obesidade }(\mathrm{n}= \\
34)\end{array}$ & Valor de $\mathrm{p}$ \\
\hline $\begin{array}{c}\text { Qualidade subjetiva do } \\
\text { sono }\end{array}$ & 0 & 1 & 1 & 0,23 \\
\hline Latência do sono & 0 & 0 & 1 & 0,14 \\
\hline $\begin{array}{c}\text { Duração do sono } \\
\text { Eficiência habitual do } \\
\text { sono }\end{array}$ & 0 & 0 & 2 & 0,02 \\
\hline Distúrbio do sono & 1 & 1 & 1 & 0,25 \\
\hline $\begin{array}{c}\text { Uso de medicação para } \\
\text { dormir }\end{array}$ & 0 & 1 & 2 & 0,01 \\
\hline Disfunção diurna & 1 & 1 & 1 & 0,18 \\
\hline Pontuação geral & 2 & 1 & 2 & 0,04 \\
\hline
\end{tabular}

\section{Discussão}

Nosso estudo mostrou que a qualidade do sono é pior em pacientes com obesidade, pois eles pontuaram mais alto do que outros grupos. O grupo eutrófico apresentou escore baixo, mostrando que os pacientes nesta faixa de IMC apresentam melhor qualidade de sono. O grupo com excesso de peso teve pontuação intermediária entre os outros dois, tendo sono prejudicado em relação ao grupo eutrófico.

Assim, constatamos que ao comparar o escore total entre os grupos estudados, pode-se perceber que a qualidade do sono tende a piorar com o aumento do IMC. Achado semelhante é mostrado no estudo de Araújo et al. ${ }^{18}$, no qual os escores de duração do sono e disfunção diurna em obesos foram estatisticamente significativos, contribuindo diretamente para o aumento do escore total do PSQI, que representou sono insatisfatório.

Embora exista uma escassez de estudos na literatura que avaliem a qualidade do sono em pacientes pós-cirurgia cardíaca, em diferentes índices de massa corporal, a fim de corroborar nossos achados, as evidências científicas a seguir revelam que a qualidade do sono pode ser influenciada pelo IMC.

Conforme mostrado na literatura, existe uma correlação entre a qualidade do sono e a cirurgia cardíaca. Bornhausen et al. ${ }^{19}$ investigaram a qualidade subjetiva do sono em pacientes com cardiopatia isquêmica crônica e identificaram $60 \%$ dos pacientes com sono ruim e obesos de acordo com o escore do questionário de Pittsburgh. Da mesma forma, nos resultados do estudo de Beck et al.20, evidenciou-se que os pacientes submetidos à cirurgia cardíaca apresentam elevada frequência de sonolência diurna excessiva, demonstrando alterações na qualidade do sono, conforme demonstrado neste estudo.

Esse aumento da sonolência diurna pós-operatória, segundo Dianatkhah et al.21, deve-se à redução da secreção de melatonina nesse período. Navarro-García et al. 2 mostraram que a qualidade do sono em pacientes submetidos à cirurgia cardíaca foi prejudicada por fatores como dor, drenagem e desconforto causados por ruídos de alarmes, aparelhos de monitoramento e vozes de funcionários de UTI.

Paralelamente, existem vários estudos científicos que revelam uma correlação entre o IMC e a qualidade do sono, mostrando que a massa corporal é um parâmetro que por si só pode influenciar na qualidade do sono dos pacientes independentemente dos procedimentos cirúrgicos. Chem et al..22 mostraram que o padrão de sono inadequado (dormir menos de 6 a 8 horas por noite) estava associado à obesidade. Para ele, a redução das horas de sono é causada pelo aumento da atividade simpática, aumento da secreção de cortisona e diminuição da intolerância à glicose. 
Segundo Cournot et al. $\underline{23}$, indivíduos obesos e menos ativos fisicamente precisam de menos sono, o que explica a alta prevalência de distúrbios do sono nessa faixa de IMC. Segundo Rahe et al. .4 , o excesso de peso pode levar à redução da atividade física durante o dia, diminuindo a vontade de dormir. Uma vez que pacientes obesos tendem a ser menos ativos, a queima calórica e o gasto energético são menores, reduzindo a restauração desse armazenamento que ocorre durante o sono. Além disso, para Driver et al. $\underline{25}$, o exercício, aumentando a temperatura corporal, criaria uma condição capaz de facilitar o desencadeamento do início do sono, ao ativar os processos de dissipação de calor controlados pelo hipotálamo.

Dadas as evidências anteriores que explicam como o IMC pode influenciar o sono, esses achados podem estar associados a altos escores na duração do sono, distúrbios do sono e disfunção diurna em pacientes obesos. Pacientes obesos têm dificuldade em iniciar ou manter o sono. É possível que a redução da necessidade de sono reduza a duração do sono, o que explica o maior escore de duração do sono para pacientes obesos. Além disso, a obesidade pode causar alterações no controle respiratório, deixando o sono instável e aumentando os despertares noturnos, o que pode explicar o maior escore da variável distúrbio do sono nessa faixa de IMC. Consequentemente, o acúmulo de noites não restauradoras, em longo prazo, pode causar redução no desempenho das atividades diárias do indivíduo, levando à sonolência diurna excessiva, justificando a mudança na variável disfunção diurna. Esses efeitos geralmente não afetam pacientes eutróficos ou com sobrepeso.

A avaliação da qualidade do sono dos pacientes no período pós-operatório, realizada no presente estudo, pode ser útil para a tomada de decisão relacionada ao tratamento fisioterapêutico, uma vez que é possível utilizar os resultados desta avaliação para orientar a criação de protocolos individualizados no pré-operatório desses pacientes de acordo com o IMC.

As limitações deste estudo devem-se principalmente à falta de estratificação por gênero dos grupos eutrófico, com sobrepeso e obesidade, o que impossibilita saber como os diferentes índices de massa corporal influenciam na qualidade do sono apenas em homens ou mulheres.

\section{Conlusão}

Com base nos achados, conclui-se que os pacientes que apresentaram maior índice de massa corporal apresentam pior qualidade do sono. Portanto, pacientes obesos tiveram pior qualidade de sono, enquanto pacientes eutróficos tiveram melhor qualidade de sono.

\section{Contribuições dos autores}

Santos ALC, Silva ALP e Brandão GM participaram da concepção e desenho da pesquisa, obtenção de dados e redação do manuscrito. Cordeiro ALL participou da concepção e desenho da pesquisa, obtenção de dados, redação do manuscrito e revisão crítica do manuscrito quanto ao conteúdo intelectual importante. Guimarães AR participou da revisão crítica do manuscrito quanto ao conteúdo intelectual importante.

\section{Conflito de interesses}

Nenhum interesse financeiro, jurídico ou político conflitante com terceiros (governo, comercial, fundação privada, etc.) foi divulgado para qualquer aspecto do trabalho submetido (incluindo, mas não se limitando a, doações, conselho de monitoramento de dados, desenho do estudo, preparação do manuscrito, estatística análise, etc.).

\section{Referências}

1. Dessotte CAM, Rodrigues HF, Furuya RK, Rossi LA, Dantas RAS. Estressores percebidos por pacientes no pós-operatório imediato de cirurgia cardíaca. Rev Bras Enferm. 2016;69(4):741-50. https:// doi.org/10.1590/0034-7167.2016690418i

2. Navarro-García MÁ, Carlos Alegre V, Martinez-Oroz A, IrigoyenAristorena MI, Elizondo-Sotro A, Indurain-Fernández S, et al. Quality of sleep in patients undergoing cardiac surgery during the postoperative period in intensive care. Enferm Intensiva. 2017;28(3):114-24. https://doi.org/10.1016/j.enfie.2017.03.002

3. Elliott R, McKinley S, Cistulli P, Fien M. Characterisation of sleep in intensive care using 24-hour polysomnography: anobservational study. Crit Care. 2013;17(2):R46. https://doi. org/10.1186/cc12565

4. Lusk B, Lash AA. The Stress Response,

Psychoneuroimmunology, and Stress Among ICU Patients.

Dimens Crit Care Nurs. 2005;24(1):25-31. https://doi. org/10.1097/00003465-200501000-00004 
5. Melo RPR, Costa ACLF, Nascimento RKG, Jesus RLR, Fortes JVS, Medeiros DD, et al. Qualidade do sono de pacientes no pósoperatório de cirurgia cardíaca. Braz J Surg Clin Res [Internet]. 2019;25(1):7-12. Disponível em: https://www.mastereditora.com. br/periodico/20181204_202822.pdf

6. Tavares SM. Guia Einsten para portadores de deficiência visual: a importância do sono. São Paulo: Instituto de ensino e pesquisa Albert Einsten; 2002.

7. Dianatkhah $M$, Ghaeli $P$, Talasaz AH, Karimi A, Salehiomran $A$, Bina $P$, et al. Evaluating the potential effect of melatonin on the post- cardiac surgery sleep disorder. J Tehran Heart Cent. 2015;10(3):122-8. Citado em: PMID: 26697084

8. Buysse DJ, Reynolds CF, Monk TH, Berman SR, Kupfer DJ. The Pittsburgh sleep quality index: A new instrument for psychiatric practice and research. Psychiatry Res. 1989;28(2):193-213. https:// doi.org/10.1016/0165-1781(89)90047-4

9. Buysse DJ, Hall ML, Strollo PJ, Kamarck TW, Owens J, Lee L, et al. Relationships between the Pittsburgh Sleep Quality Index (PSQI), Epworth Sleepiness Scale (ESS), and clinical/polysomnographic measures in a community sample. J Clin Sleep Med. 2008;4(6):56371. Citado em: PMID: 19110886

10. Cole J, Motivala S, Buysse D, Oxman M, Levin M, Irwin M. Validation of a 3-Factor Scoring Model for the Pittsburgh Sleep Quality Index in Older Adults. Sleep. 2006;29:112-6. https://doi. org/10.1093/sleep/29.1.112

11. Vargas PA, Flores M, Robles E. Sleep Quality and Body Mass Index in College Students: The Role of Sleep Disturbances. J Am Coll Health. 2014;62(8):534-41. https://dx.doi. org/10.1080\%2F07448481.2014.933344

12. Ardani AR, Talaei A, Moghani MB, Nejati R, Sabouri S, Solooti $S$, et al. Assessment the rules of demographic variables and body mass index in sleep quality among medical students. J Fund Ment Health. 2012;14(2):132-9. https://dx.doi.org/10.22038/ jfmh.2012.983

13. Yeh S-SS, Brown RF. Disordered eating partly mediates the relationship between poor sleep quality and high body mass index. Eating Behaviors. 2014;15(2):291-7. https://doi. org/10.1016/j.eatbeh.2014.03.014

14. Gupta NK, Mueller WH, Chan W, Meininger JC. Is obesity associated with poor sleep quality in adolescents?. Am J Hum Biol. 2002;14(6):762-8. https://doi.org/10.1002/ajhb.10093

15. Shochat T, Shefer-Hilel G, Zisberg A. Relationships between body mass index and sleep quality and duration in adults 70 years and older. Sleep Health. 2016;2(4):266-71. https://doi. org/10.1016/j.sleh.2016.09.001
16. Madrid-Valero JJ, Martínez-Selva JM, Ordoñana JR. Sleep quality and body mass index: a co-twin study. J Sleep Res. 2017;26(4):4617. https://doi.org/10.1111/jsr.12493

17. Hung HC, Yang YC, Ou HY, Wu JS, Lu FH, Chang CJ. The association between self-reported sleep quality and overweight in a Chinese population. Obesity (Silver Spring). 2013;21(3):486-92. https://doi.org/10.1002/oby.20259

18. Araujo PAB, Sties SW, Wittkopf PG, Netto AS, Gonzáles AI, Lima DP, et al. Índice da Qualidade do Sono de Pittsburgh para uso na reabilitação cardiopulmonar e metabólica. Rev Bras Med Esporte. 2015;21(6):472-5. http://dx.doi.org/10.1590/1517$\underline{869220152106147561}$

19. Bornhausen A, Kessler RMG, Gasperin SI. Qualidade subjetiva do sono em cardiopatas isquêmicos crônicos. Insuf Card [Internet]. 2018;13(3):110-7. Disponível em: https://docs.bvsalud. org/biblioref/2018/09/914519/v13n3a03.pdf

20. Back GD, Goulart CL, Antunes-San-Martin E, Schneiders PB, Hippler A, Ourives-Barbosa R, et al. Frequência de sonolência diurna e apneia obstrutiva do sono em pacientes submetidos à cirurgia cardíaca participantes de um Programa de Reabilitação Cardiorrespiratória. Revista Jovens Pesquisadores. 2016;6(1):4557. http://dx.doi.org/10.17058/rjp.v6i1.7290

21. Dianatkhah M, Ghaeli $P$, Hajhossein Talasaz A, Karimi A, Salehiomran A, Bina $P$, et al. Evaluating the Potential Effect of Melatonin on the post-Cardiac Surgery Sleep Disorder. J Tehran Heart Cent. 2015;10(3):122-8. Citado em: PMID: 26697084

22. Chen M-Y, Wang EK, Jeng Y-J. Adequate sleep among adolescents is positively associated with health status and healthrelated behaviors. BMC Public Health. 2006;6:59. https://doi. org/10.1186/1471-2458-6-59

23. Cournot M, Ruidavets JB, Marquié JC, Esquirol Y, Baracat B, Ferrières J. Environmental factors associated with body mass index in a population of Southern France. Eur J Cardiovasc Prev Rehabil. 2004;11(4):291-7. https://doi.org/10.1097/01. hjr.0000129738.22970.62

24. Rahe C, Czira ME, Teismann H, Berger K. Associations between poor sleep quality and different measures of obesity. Sleep Med. 2015;16(10):1225-8. https://doi.org/10.1016/j.sleep.2015.05.023

25. Driver HS, Taylor SR. Exercise and sleep. Sleep Med Rev. 2000;4(4):387-402. https://doi.org/10.1053/smrv.2000.0110 